\title{
Age influences domestic dog cognitive performance independent of average breed lifespan
}

Marina M. Watowich ${ }^{1}$, Evan L. MacLean ${ }^{2,3,4}$, Brian Hare $^{5,6}$, Josep Call ${ }^{7}$, Juliane Kaminski ${ }^{8}$, Ádám Miklósi ${ }^{9,10}$, Noah Snyder-Mackler ${ }^{1,11,12,13,14,15}$

Correspondence: Noah Snyder-Mackler, nsnyderm@asu.edu

${ }^{1}$ Department of Biology, University of Washington, Seattle, WA 98195, USA

${ }^{2}$ School of Anthropology, University of Arizona, Tucson, AZ 85719, USA

${ }^{3}$ Department of Psychology, University of Arizona, Tucson, AZ 85721, USA

${ }^{4}$ Cognitive Science, University of Arizona, Tucson, AZ 85721, USA

${ }^{5}$ Department of Evolutionary Anthropology, Duke University, Durham, NC 27708, USA

${ }^{6}$ Center for Cognitive Neuroscience, Duke University, Durham, NC 27708, USA

${ }^{7}$ School of Psychology and Neuroscience, University of St Andrews, St Andrews, UK

${ }^{8}$ Centre for Comparative and Evolutionary Psychology, Department of Psychology, University of Portsmouth, Portsmouth, UK

${ }^{9}$ Department of Ethology, Eötvös Loránd University, Budapest, Hungary

${ }^{10}$ MTA-ELTE Comparative Ethology Research Group, Budapest, Hungary

${ }^{11}$ Center for Evolution \& Medicine, Arizona State University, Tempe, AZ 85287, USA

${ }^{12}$ School of Life Sciences, Arizona State University, Tempe, AZ 85287, USA

${ }^{13}$ Department of Psychology, University of Washington, Seattle, WA 98195, USA

${ }^{14}$ Nathan Shock Center of Excellence in the Basic Biology of Aging, Department of Pathology, University of Washington, Seattle, WA 98195, USA

${ }^{15}$ Center for Studies in Demography and Ecology, University of Washington, Seattle, WA 98195, USA

Abstract

Across mammals, increased body size is positively associated with lifespan. However, within species, this relationship is inverted. This is well illustrated in dogs (Canis familiaris), where larger dogs exhibit accelerated life trajectories: growing faster and dying younger than smaller dogs. Similarly, some age-associated traits (e.g., growth rate and physiological pace of aging) exhibit accelerated trajectories in larger breeds. Yet, it is unknown whether cognitive performance also demonstrates an accelerated life course trajectory in larger dogs. Here, we measured cognitive development and aging in a cross-sectional study of over 4000 dogs from 66 breeds using nine memory and decision-making tasks performed by citizen scientists as part of the Dognition project. Specifically, we tested whether cognitive traits follow a compressed (accelerated) trajectory in larger dogs, or the same trajectory for all breeds, which would result in limited cognitive decline in larger breeds. We found that all breeds, regardless of size or lifespan, tended to follow the same quadratic trajectory of cognitive aging — with a period of cognitive

36 development in early life and decline in later life. Taken together, our results suggest that cognitive performance

37 follows similar age-related trajectories across dog breeds, despite remarkable variation in developmental rates and 38 lifespan. 
41 Cognitive evolution, Cognitive aging, Breed differences, Citizen science, Executive function 42 
Introduction

Across mammals, larger species tend to live longer than smaller species (Healy et al. 2014). Yet, within species this pattern is reversed (Metcalfe and Monaghan 2003; Austad 2010; Bartke 2017). This pattern is well-

46 documented in domestic dogs (Galis et al. 2007; Kraus et al. 2013; Fan et al. 2016) where larger dog breeds (e.g.,

47 Bernese Mountain Dog, mean lifespan $=7$ years) have an expected lifespan that is approximately half that of smaller breeds (e.g., Chihuahua, mean lifespan $=13$ years; Jones et al. 2008). While large breeds take longer to mature than small breeds, they weigh disproportionately more, and therefore have faster growth rates and an accelerated pace of physiological aging (e.g., cellular damage; Fick et al. 2012; Kraus et al. 2013; Fan et al. 2016). Domestic dogs have been under strong artificial selection for at least 15,000 years (vonHoldt et al. 2010), which has driven extensive diversity in physical and life history traits (i.e., size, growth rate, lifespan). The large variation in these life history traits, in particular, have made dogs an invaluable model species for studying the underpinnings of age-related changes in health (Hoffman et al. 2018).

Domestic dogs also provide a powerful model in which to explore intraspecific patterns of cognitive aging. In humans and other animals, including dogs, cognitive abilities, such as learning and memory, change throughout aging (Craik and Bialystok 2006; Bizon and Woods 2009; Harada et al. 2013; Chapagain et al. 2018). However, for some cognitive processes, dogs may even provide a better model for human cognition than rodents and nonhuman

59 primates, potentially due to convergent evolution between humans and dogs (Miklósi et al. 2004; Hare and

60 Tomasello 2005; Hare 2017; MacLean et al. 2017). For millennia, humans have selected dogs for both behavioral

61 (i.e., herding, hunting) and physical traits (Ostrander et al. 2017; Parker et al. 2017), contributing to the extensive

62 diversity seen across modern breeds. While the association between domestic dog cognition and other life history

63 traits (e.g., age) remains largely unexplored, there is evidence that absolute brain size is associated with breed

64 differences in executive function (Horschler et al. 2019) - a cognitive domain responsible for inhibitory control,

65 mental flexibility, and decision-making (Alvarez and Emory 2006; Jurado and Rosselli 2007).

67 and cognitive flexibility (Diamond 2013). In humans, executive function, learning, and long-term memory have

68 largely been found to increase in early life and decrease in late life (Craik and Bialystok 2006; Harada et al. 2013;

69 but see Verhaeghen 2011). Other cognitive abilities (e.g., vocabulary and general knowledge) increase steadily

70 throughout life (i.e., linearly) or increase rapidly and then plateau (i.e., resembling a positive logarithmic curve) 
71 (Harada et al. 2013). Similar to humans, domestic dogs experience changes to critical cognitive functions across life

72 (Chapagain et al. 2018). Recent studies from laboratory and pet dogs have demonstrated that learning, memory, and

73 cognitive functions under executive control decrease in older dogs (Adams et al. 2000; Tapp et al. 2003; Szabó et al.

74 2016; Wallis et al. 2016). Older dogs also show greater variability in the extent of cognitive decline with age

75 (Adams et al. 2000). One source of this variability may be lifelong behavioral training, which has been associated

76 with greater sustained and selective attention in older dogs (Chapagain et al. 2017). Although domestic dogs exhibit

77 age-related cognitive changes, we still know very little about how cognition changes with age, in large part due to

78 sample size: collecting data from enough very young and very old dogs has been challenging (Szabó et al. 2016). To

79 date, most studies of dog cognitive aging have focused on one breed or a small number of breeds, which, due to the

80 limited variability in life history within individual breeds, has limited our ability to examine associations between

81 cognitive aging and physiological pace of aging. Consequently, the extent to which cognitive changes throughout

82 aging are associated with life history traits that covary with physiological pace of aging (as measured by average

83 breed lifespan) remains unknown.

84 Here, we investigated the associations between lifespan and cognitive traits in dogs, in order to test the

85 hypothesis that animals with faster life histories also exhibit earlier onset of and/or more rapid cognitive decline. We

86 addressed two questions using a cross-sectional dataset of more than 4,000 dogs from 66 breeds collected from

87 participants of the Dognition project, a citizen-science initiative in which owners perform simple cognitive tests with

88 their dogs at home (Stewart et al. 2015). First, we tested how cognition changes across the lifespan of domestic

89 dogs. To date, many studies of dog cognition have modeled cognition as a linear process throughout the lifespan, yet

90 data from humans, apes, and several within-breed studies of domestic dogs suggest the likelihood of non-linear

91 changes across development and senescence (Craik and Bialystok 2006; Harada et al. 2013; Wallis et al. 2014;

92 Manrique and Call 2015). Second, we tested if and how a key life history trait, expected breed lifespan, affected the

93 trajectory of cognition across the lifespan. We explicitly tested two alternative hypotheses: i) truncation: that all

94 breeds have similar cognitive trajectories throughout aging with larger breeds having a limited period of cognitive

95 decline (Fig. 1a), and ii) compression: that changes in cognitive abilities scale with lifespan such that larger dogs

96 have a compressed (i.e., accelerated) cognitive trajectory (Fig. 1b). 


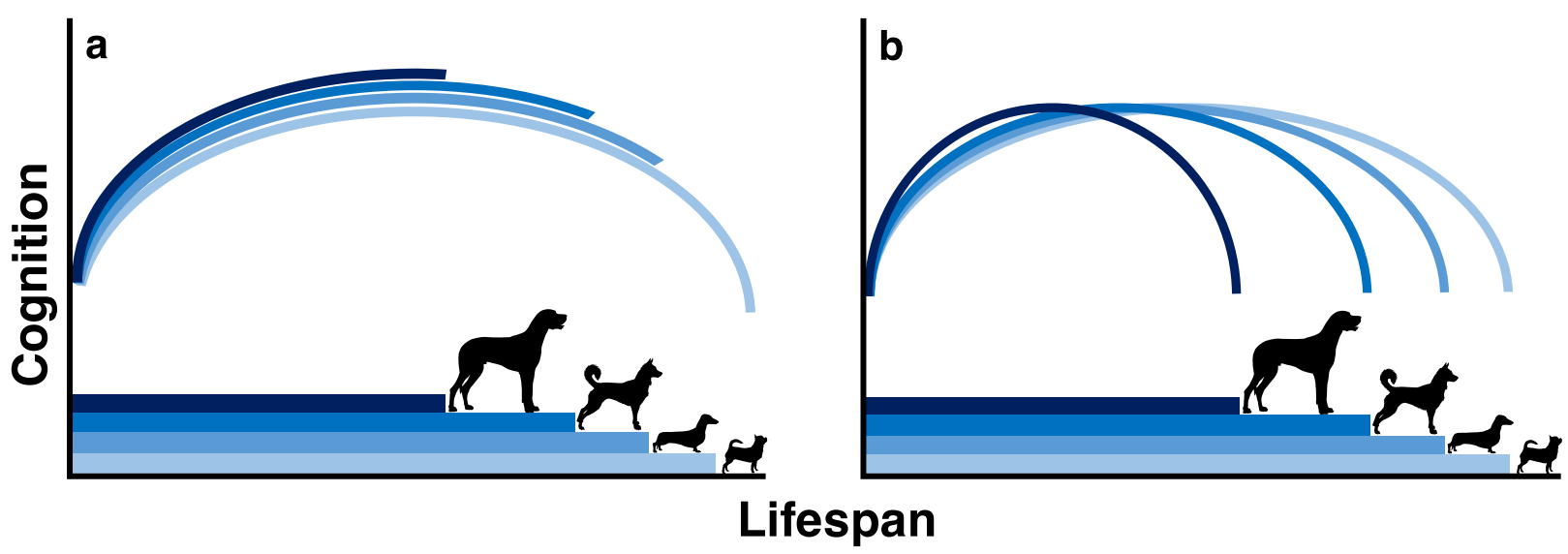

98 Fig. 1 Alternative models of cognitive aging in dogs. a Schematic of the truncation hypothesis in which larger and

99 smaller dog breeds have similar cognitive trajectories throughout aging. Under this hypothesis, large dog breeds

100 experience limited cognitive decline because they typically die before the more precipitous cognitive decline

101 experienced by longer-lived, smaller breeds. b The compression hypothesis in which cognitive performance scales

102 with lifespan, such that larger breeds will have an accelerated cognitive trajectory.

\section{Methods}

105 Data sources

106 Cognitive performance data were collected from Dognition.com, a citizen science website which provides 107 users with video instructions for completing simple cognitive experiments at home with their dogs. Owners entered 108 data into the website by answering simple questions about their dog's behavior during the cognitive tests (e.g., 109 which location did your dog approach?). Importantly, results from citizen scientists using Dognition recapitulate 110 results from professional scientists working in controlled laboratory settings (Stewart et al. 2015). Here, we 111 restricted our analysis to data collected prior to April 2018 from purebred dogs with known sex, age, reproductive 112 alteration status (i.e., spayed/neutered vs. intact), and breed $(n=4,419)$. Dogs in the study represented 66 breeds and 113 ranged in age from $<1$ to 14.2 years with a mean age of 4.78 years (standard deviation \pm 3.13 ; Supplemental Fig. 1).

114 To ensure representative sampling, only breeds with 10 or more individuals were retained for analysis

115 (Supplemental Table 1). We used data from purebred dogs to ensure that we could estimate mean breed lifespan and 116 to control for relatedness among breeds based on breed-averaged genotypic data (Parker et al. 2017; Supplemental 117 Table 2). We used estimates of mean breed lifespan from Jones et al. (2008; Supplemental Table 3). Because recent 
118 studies have found behavioral modifications correlated with reproductive alteration (Hart 2001; Mongillo et al.

119 2017; Scandurra et al. 2019), we also included reproductive alteration status as a covariate in all models.

120 We included data from nine cognitive tasks measuring diverse processes involving aspects of executive

121 function, such as memory, reasoning, decision making, self-control, as well as measures of social cognition (Stewart

122 et al. 2015; Horschler et al. 2019; Table 1). Sample size varied across tasks due to participant attrition across the

123 series of experiments. We focused our analyses on tasks involving executive function as it is one of the cognitive

124 domains most susceptible to effects of aging (Jurado and Rosselli 2007). Seven of the Dognition tasks involved an

125 object-choice paradigm in which the dog had to choose one of two possible options, across a series of trials

126 (range=1-6, mode=4; Table 1). One of the other two tasks was the eye contact task, in which the owner held a piece

127 of food up to their face and recorded the time until the dog broke eye contact (up to 90 seconds). This task was

128 designed as a measure of dog's social engagement. In the remaining task, the owner set a treat before the dog,

129 instructed the dog not to take a treat, and recorded the time until the dog took the treat (up to 90 seconds) while the

130 owner i) was visibly watching the dog, ii) had their back turned to the dog, or iii) faced the dog with their eyes

131 closed (detailed in Stewart et al. 2015). Although originally developed as a measure of social cognition (sensitivity

132 to cues about the human's visual perspective), recent analyses have focused on the executive function component of

133 this task, which requires dogs to delay gratification (Horschler et al. 2019). Following this approach, we considered

134 the latency to take the forbidden treat as a measure of executive function in our analyses. To summarize

135 performance in this task, we performed a principal component analysis on the latencies to take the treat across

136 conditions. This analysis yielded one principal component which explained $88 \%$ of the variance and is subsequently

137 referred to as 'delay of gratification'. The Dognition battery also includes a contagious yawning task which we did

138 not include because preliminary analyses showed minimal evidence for contagious yawning in this sample.

Table 1 Description of cognitive tasks, cognitive processes the task was designed to test, number of trials conducted per task, number of individuals included in analysis, and number of breeds included in analysis

Task Description

Eye contact if and when the dog breaks eye contact within 90 seconds.

\section{Cognitive processes $\quad$ Trials $\quad \begin{gathered}\text { Total } \\ \text { dogs }\end{gathered} \begin{gathered}\text { Total } \\ \text { breeds }\end{gathered}$}

Social engagement $\quad 3 \quad 4359 \quad 65$ 
Arm pointing

Foot pointing

Delay of gratification Watching verbally commands the dog not to take the treat. condition The owner records the duration of time until the dog takes the treat, up to 90 seconds.

Closed eyes condition

Same as above, with the owner closing their eyes.

Turned back condition

Memory vs. pointing

Memory vs. smell

Delayed memory

Inferential reasoning

Physical reasoning

142 points to one treat location, and records the location the dog first approaches.

The owner places one treat to their right and left, extends their foot toward one treat location, and records the location the dog first approaches.

Same as above, with the owner turning their back.

In view of the dog, the owner places a treat under one of two cups, then proceeds to point to the other cup. The owner records which location the dog first approaches.

Allowing the dog to see, the owner places a treat under one of two cups, then blocks the dog's view while switching the position of the treat. The owner records which location the dog first approaches.

In full view of the dog, the owner places a treat under one of two cups and then waits 60, 90, 120, and 150 seconds (across four trials) before releasing the dog. Then the owner records which location the dog first approaches.

The owner appears to place treats under two cups, while only baiting one. The owner raises the empty cup to show it is empty and records which location the dog first approaches.

Blocking the dog's view, the owner places two pieces of folded paper on the floor. The owner places a treat under one paper so that the paper is elevated by the treat while the other paper is flat and records which location the dog first approaches.
The owner places one treat to their right and left,
Social cognition/

communication

6

4071

63

Inhibition/self-control

6

2826 51

Social cognition/

4367

65

Inhibition/self-control

$2 \quad 2826$

51

2826

51

2826

51

Bias for information from memory vs. communication

$6 \quad 2346$

Bias for information from memory vs. olfaction

Short-term memory/ sustained attention

4 2124 47

Inferential reasoning/ reasoning by

exclusion

Physical causality/ inferential reasoning 
To address the questions of i) how cognitive performance changes across domestic dog lifespan (Equations

1451,2 , and 3) and ii) whether cognition scales with mean breed lifespan (Equations 3 and 4), we compared the fit of all

146 four mixed effects models for each of the seven tasks with a binomial response and ii) the two linear response

147 variables (eye contact and the principal component scores reflecting delay of gratification; Supplemental Tables 4,

148 5). Because many cognitive abilities in humans exhibit a negative quadratic relationship (an inverted U-shape) with

149 age (in particular those associated with executive processes), while others tend to increase throughout life or

150 increase quickly during development and then plateau (Craik and Bialystok 2006; Harada et al. 2013; Wallis et al.

151 2014), we tested if cognitive performance followed these trajectories by modeling age as a quadratic, linear, and

152 logarithmic predictor (Supplemental Table 4).

(1) Linear trajectory: Cognitive measure $\sim$ sex + reproductive alteration + age + mean breed lifespan

(2) Logarithmic trajectory: Cognitive measure $\sim$ sex + reproductive alteration $+\log (\operatorname{age})+$ mean breed lifespan

(3) Quadratic trajectory (additive): Cognitive measure $\sim$ sex + reproductive alteration + age + age ${ }^{2}+$ mean breed lifespan

(4) Quadratic trajectory (interactive): Cognitive measure $\sim$ sex + reproductive alteration + age $\times$ mean breed lifespan + age $e^{2} \times$ mean breed lifespan

Equations 1, 2, 3, and 4 Equations 1, 2, and 3 represent hypothesized trajectories of cognitive aging, based on previously described patterns of cognitive aging. Equations 3 and 4 represent the truncation and compression hypotheses

In all models, the age and mean breed lifespan predictors were mean centered and scaled to a standard

160 than the predictors of sex and reproductive alteration. Scaling the predictors of age and mean breed lifespan made

161 them similar in magnitude to the predictors of sex and reproductive alteration, facilitating interpretation of the model

162 results (Harrison et al. 2018). All models of the quadratic trajectory included orthogonal linear and quadratic

163 predictors of age. Mixed-effects models of the seven binomial measures were carried out using the 'PQLseq'

164 package, which implements the mixed modeling framework MACAU in the R environment (Lea et al. 2015; Sun et

165 al. 2019). This allowed us to model a binomial outcome variable (i.e., number of times dog chose the left cup out of

166 6) while controlling for background genetic similarity among the breeds, which was calculated from a recent

167 genomic analysis of 150,067 SNPs in 1,346 dogs representing 161 breeds (Parker et al. 2017; Supplemental Table

168 2). The eye contact and delay of gratification measures were modeled controlling for breed relatedness using the 
'EMMREML' package (Akdemir and Okeke 2015) in the R environment. Only breeds for which we had both cognitive and genetic data were included in our analyses (Supplemental Table 1). Model fits were compared using

171 Akaike information criterion (AIC; Akaike 1974). To test the sensitivity of our models, we also repeated these

172 analyses excluding overrepresented breeds, which were breeds which each constituted over $5 \%$ of the dataset

173 (Supplemental Table 6). Five breeds fit this criterion (Australian Shepherds, Border Collies, German Shepherd

174 Dogs, Golden Retrievers, and Labrador Retrievers) and together comprised over $45 \%$ of the dataset. For models of

175 the three tasks involving gesture following (arm pointing, foot pointing, and memory vs. pointing), we included

176 behavioral training history as a predictor in the truncation and compression models to ensure that our results were

177 not confounded by the correlation between dog size and training history (training history was rated on a scale of 1

178 [none] -4 [substantial]; $\mathrm{r}_{\mathrm{s}}=0.12, \mathrm{p}<0.001$; Supplemental Table 7; Horschler et al. 2019).

\section{Delayed memory task}

We considered the delayed memory task the clearest test of basic memory because other tests involving

182 memory measured preferences and biases, where memory was pitted against other information sources (e.g.,

183 memory of treat location vs. owner's pointing, memory of treat location vs. scent of the treat). Although designed

184 and interpreted as a measure of memory, it is possible that the delayed memory task also reflects variation in

185 sustained attention, since the hiding locations were not out of the dog's view during the delay. However, unlike

186 traditional sustained attention tasks, there was no cue provided at the end of the delay, and thus subjects would most

187 likely have been reliant on memory to motivate their search for food at the baited location. We modeled all trials

188 from this task to test the truncation and compression hypotheses, however, we focused our analyses on the 150

189 second trial as this trial was the longest time delay and likely the most cognitively challenging. Importantly, the

190 results of the 60,90 , and 120 second delayed memory trials were very similar to the 150 second trial (Supplemental

191 Table 8).

192

\section{Results}

\section{Cognition across the lifespan}

195 For all tasks, the best models $(\Delta \mathrm{AIC}>2)$ included the quadratic predictor of age, compared to linear or 196 logarithmic, meaning that cognitive performance followed the expected inverted U-shaped trajectory - increasing in 
197 early life and declining in late life (Figs. 2, 3; Supplemental Fig. 2; Supplemental Table 4). Although the best

198 models included quadratic functions of age, the coefficient for the quadratic term was significant in only six tasks

199 (eye contact, delayed memory, memory vs. pointing, memory vs. smell, and inferential reasoning tasks, as well as

200 the delay of gratification score; Fig. 2, Fig. 3; Supplemental Tables 4, 5, 8). Models without a significant age ${ }^{2}$ term

201 (arm pointing, foot pointing, and physical reasoning tasks) showed an increase in cognitive performance throughout

202 aging (Supplemental Table 5).

203

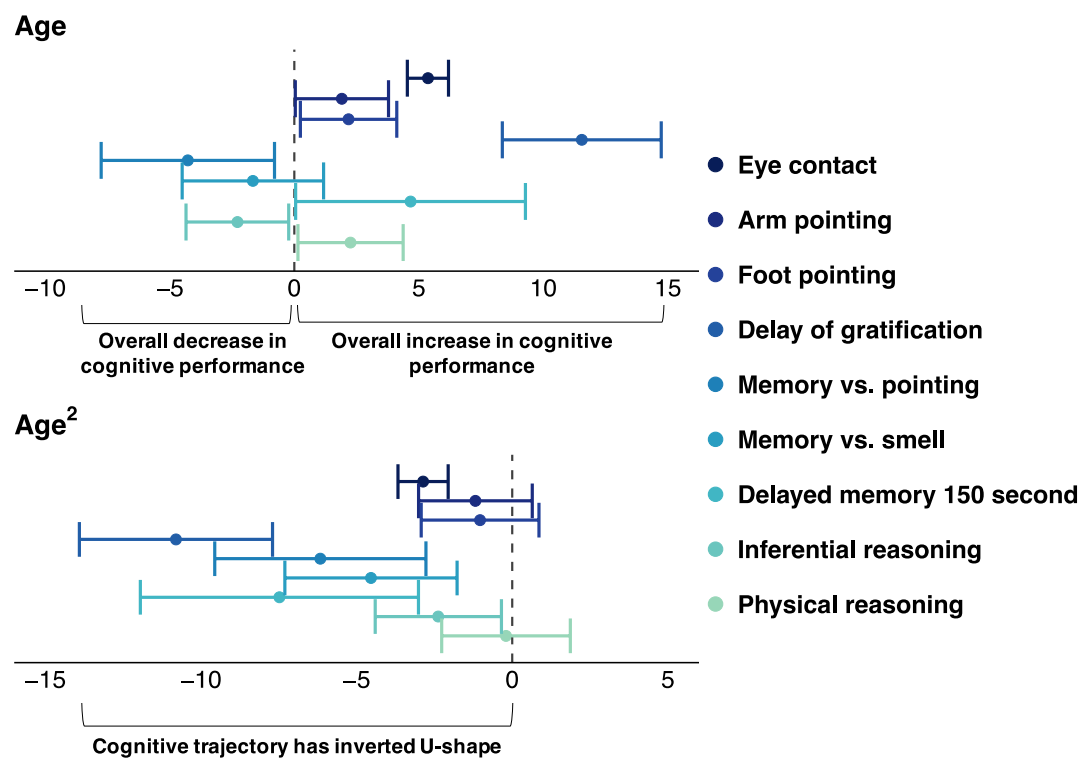

Fig. 2 Age has both linear and quadratic associations with cognitive function. Effect sizes and estimated

206 confidence intervals (beta $\pm 1.96 \times S E$ ) of age and age $^{2}$ predictor variables for the additive (truncation) models for

207 each cognitive task. Estimated confidence intervals that do not cross the line of null effect $(\mathrm{x}=0)$ are statistically

208 significant. For ease of interpretation and visualization, and to keep all variables on a similar scale, we converted the 209 beta for the eye contact task to minutes 


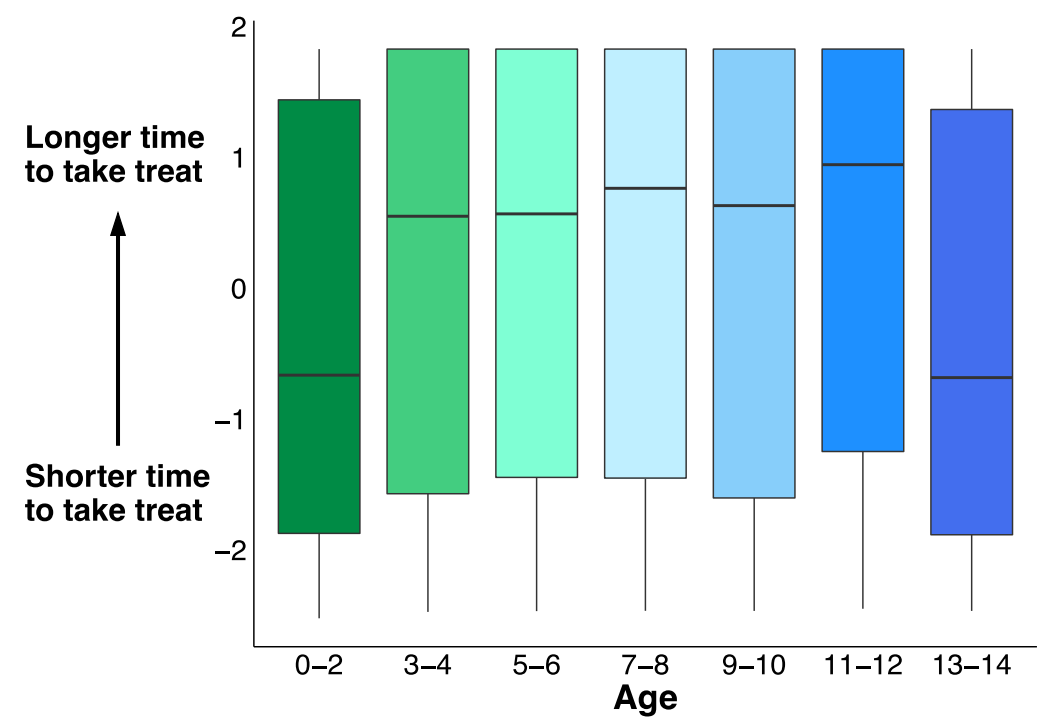

212 Fig. 3 Self-control changes with age. Delay of gratification principal component 1 values for 2-year age groups.

213 The delay of gratification task measures the time until the dog takes a treat (latency) under conditions of the owner

214 watching, closing their eyes, and turning their back. Increasing y-axis values indicate greater performance in

215 prolonging gratification (i.e., increased latency to take the treat)

217 Truncation vs. compression models

218 Models testing the truncation hypothesis fit the data better than or as well as models of the compression

219 hypothesis for seven of the nine tasks (Table 2; Supplemental Table 5). The two tasks for which the model of the

220 compression hypothesis had a better model fit were the eye contact task and delay of gratification score. However,

221 neither of these models had significant interactions with mean breed lifespan, between age and mean breed lifespan,

222 or between $\mathrm{age}^{2}$ and mean breed lifespan. For models of the compression hypothesis, only the arm pointing ( $\beta=$

$2232.196, \mathrm{SE}=0.996, \mathrm{p}=0.027, \mathrm{n}=4367)$ and foot pointing tasks $(\beta=2.017, \mathrm{SE}=1.028, \mathrm{p}=0.049, \mathrm{n}=4071)$ showed

224 significant interaction effects between age ${ }^{2}$ and mean breed lifespan, while the memory vs. pointing task $(\beta=0.087$,

$225 \mathrm{SE}=0.042, \mathrm{p}=0.037, \mathrm{n}=2346$ ) had a significant effect of mean breed lifespan in models of the truncation and

226 compression hypotheses. These associations would support the compression hypothesis; however, interactive

227 models of these tasks did not fit the data better than additive models representing the truncation hypothesis $(\triangle \mathrm{AIC}<$

228 2; Table 2; Supplemental Table 5). Our sensitivity analyses that excluded overrepresented breeds generally

229 recapitulated results of the comparison between truncation and compression hypothesis models (Supplemental Table

230 6). In these analyses, fewer tasks had significant effects of age and $a^{2} e^{2}$, likely due to reduced power, however the 
231 results were very similar to analyses with the entire dataset and did not suggest that our results were being driven by

232 breeds that were overrepresented in the data. While we focused our analyses on the 150 second delayed memory

233 trial, we evaluated how age affected cognitive performance across all delayed memory trials by modeling delay-

234 specific accuracies in young (0-5 years), middle-aged (6-10 years), and old dogs (11+ years). Comparing the slope

235 of the delay function, we found that dogs 11 years and older performed lower across all delayed memory trials

236 (Supplemental Fig. 3).

Table 2 Comparing the fit of models of the truncation hypothesis and the compression hypothesis for each cognitive task. Lower AIC value indicates a better model fit. $\triangle \mathrm{AIC}>2$ is considered a difference in model fit. The numbers in bold denote a difference in model fit between truncation and compression models

$\triangle \mathrm{AIC}$ of truncation hypothesis models and compression hypothesis

\begin{tabular}{lrr}
\multicolumn{1}{c}{ Task } & models (compression - truncation) & Model with better fit \\
\hline Eye contact & $\mathbf{- 1 3 . 0}$ & compression hypothesis \\
Arm pointing & 1.2 & no difference \\
Foot pointing & -0.3 & no difference \\
Delay of gratification & $\mathbf{- 7 . 5}$ & compression hypothesis \\
Memory vs. pointing & 0.1 & no difference \\
Memory vs. smell & 0.8 & no difference \\
Delayed memory 150 second & -0.2 & no difference \\
Inferential reasoning & $\mathbf{2 . 4}$ & truncation hypothesis \\
Physical reasoning & 1.7 & no difference \\
\hline
\end{tabular}

\section{Gesture following}

243 We observed associations between predictors involving breed-mean lifespan for the three tasks involving

244 gesture following (arm pointing, foot pointing, and memory vs. pointing), supporting the compression hypothesis,

245 although model fit did not differ substantially between models of the truncation and compression hypothesis.

246 Additionally, in all models of gesture following, the age coefficient indicated increases in performance with aging,

247 rather than late-life deterioration of cognitive performance, suggesting that these associations are not likely to

248 support accelerated cognitive deterioration in faster-aging breeds. To ensure that our results were not confounded by

249 the correlation between dog size and training history, we included training as a predictor in the truncation and

250 compression models for all gesture following models. The proportion of dogs in each category of training history

251 was similar between intact and spayed and neutered individuals, males and females, and across dogs of all ages (in

252 one-year age groups). Effects of breed lifespan were reduced to statistically indistinguishable from zero when we 
included training history in the models of gesture following tasks (arm pointing [mean breed lifespan $\times$ age ${ }^{2}$ ]: $\beta$

$254=-0.64, \mathrm{SE}=0.915, \mathrm{p}=0.485, \mathrm{n}=1105$; foot pointing [mean breed lifespan $\times$ age $\left.^{2}\right]: \beta=0.275, \mathrm{SE}=0.936, \mathrm{p}=$

$2550.769, \mathrm{n}=1057$; memory vs. pointing [mean breed lifespan]: $\beta=0.071, \mathrm{SE}=0.083, \mathrm{p}=0.394 \mathrm{n}=720$;

256 Supplemental Table 7). However, because not all owners reported their dog's training history - resulting in reduced

257 power for this analysis - we could not rule out the possibility that this reduction in significance was due to a smaller

258 sample size. We therefore compared this analysis to an analysis of the same subsample excluding training history as

259 a predictor (Supplemental Table 7) and found that the effects of mean breed lifespan were not significant.

\section{Associations of reproductive alteration vary across gesture following tasks} pointing: $\beta=-0.117, \mathrm{SE}=0.039, \mathrm{p}<0.01, \mathrm{n}=4367$; memory vs. pointing: $\beta=0.379, \mathrm{SE}=0.097, \mathrm{p}<0.001, \mathrm{n}=$

264 2346; Supplemental Table 5). After controlling for training history in analyses of the arm pointing and memory vs.

265 pointing tasks, reproductive alteration was still significantly associated with decreased gesture following (arm pointing: $\beta=-0.272, \mathrm{SE}=0.073, \mathrm{p}<0.001, \mathrm{n}=1105$; memory vs. pointing: $\beta=0.527, \mathrm{SE}=0.164, \mathrm{p}<0.01, \mathrm{n}=$

267 720), indicating that spayed or neutered animals were less likely to follow social cues from their owner, even after 268 controlling for training history (Supplemental Table 7).

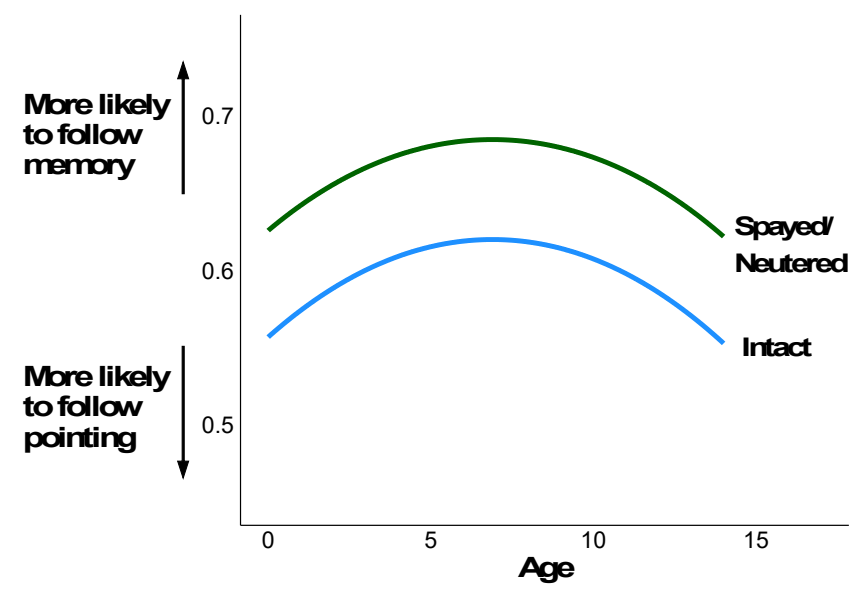

271 Fig. 4 Gesture following differs between intact and spayed and neutered dogs. Predicted cognitive trajectory for

272 the memory vs. pointing task throughout aging (years) of an average dog in the dataset whether spayed or neutered

273 (green) or intact (blue), with bootstrapped 95\% confidence intervals 


\section{Sex differences}

276 In the eye contact task, males maintained eye contact with the experimenter longer than females $(\beta=2.26$,

$277 \mathrm{SE}=0.738, \mathrm{p}=0.002, \mathrm{n}=4359$ ), but this effect size was small (males held eye contact for an average of 2.26

278 seconds longer than females). In the delayed memory task, males were more likely than females to locate a treat

279 after the 150 second delayed memory trial $(\beta=0.204, \mathrm{SE}=0.101, \mathrm{p}=0.043, \mathrm{n}=2124)$, but not any other delayed

280 memory trial; again this effect was small, but statistically significant (males had $22 \%$ higher odds of choosing the

281 cup with the treat). After performing our sensitivity analyses by excluding overrepresented breeds, males still

282 showed longer social engagement in the eye contact task $(\beta=2.323, \mathrm{SE}=1.008, \mathrm{p}=0.021, \mathrm{n}=2318)$ but sex

283 differences for the 150 second delayed memory trial were no longer significant.

\section{Discussion}

the lifespan, with most measures following a clear negative quadratic trajectory across the lifespan. For each of the

nine cognitive tasks we evaluated, models with a quadratic term for age better fit the data than linear and logarithmic terms and six of these cognitive tasks showed a distinct inverted U-shape across aging (Fig. 2; Supplemental Fig. 2;

290 Supplemental Tables 4, 5), indicating that a broad suite of cognitive processes in domestic dogs increase in early

291 life, peak in midlife, and decrease in late life. Additionally, we found that cognitive performance in tasks testing 292 physical reasoning and the propensity to follow owners' pointing gestures (without competing sources of

293 information) increase throughout aging.

294 Tasks directly testing memory and self-control were the clearest tests of executive function and had 295 particularly robust quadratic curves throughout aging (Fig. 2.; Fig. 3.; Supplemental Table 5). In humans, executive

296 function follows a similar negative quadratic trajectory throughout the lifespan and is one of the cognitive domains

297 most susceptible to aging. Declines in executive function greatly impact daily life by reducing cognitive

298 performance in domains such as decision making, memory, and self-control (Jurado and Rosselli 2007; Alvarez and

299 Emory 2006; Bizon and Woods 2009; Harada et al. 2013). Similar declines have been reported in nonhuman primate

300 and rodent models (Moore et al. 2006; Rodefer and Nguyen 2008; Beas et al. 2013), and recently described for

301 domestic dog attention (Wallis et al. 2014). Our findings extend these similarities to dogs across a range of cognitive 
processes, thus building on previous laboratory work with dogs (Milgram et al. 1994; Head et al. 2001; Tapp et al. 2003) and advancing companion dogs as a useful model for human cognitive aging.

304 To assess associations between cognition and aging, we tested models representing what we have termed 305 the 'truncation hypothesis', in which all dogs have similar cognitive trajectories, and the 'compression hypothesis', 306 in which the timing of decline is accelerated in shorter-lived breeds. Models representing the truncation hypothesis 307 fit the data better than or as well as models representing the compression hypothesis across seven of the nine 308 cognitive tasks (Table 2; Supplemental Table 5). The two tasks for which models representing the compression 309 hypothesis fit the data better than models representing the truncation hypothesis did not have significant associations 310 of mean breed lifespan or between mean breed lifespan and linear or quadratic terms of age. Additionally, all models 311 representing the compression hypothesis lacked significant interactions between age and mean breed lifespan after 312 controlling for training history. Thus, we conclude that there is not sufficient evidence for the compression 313 hypothesis, and that these results most strongly support the truncation hypothesis. Together, our results suggest that 314 all dog breeds, regardless of average breed lifespan or rate of physiological aging, exhibit similar cognitive aging 315 trajectories such that larger dogs may experience a limited cognitive decline at the end of their shorter lives.

316 Although the pace of physiological aging varies with lifespan, there is evidence that the age of onset of 317 senescence does not differ among breeds, except, perhaps, in very large breeds (Kraus et al. 2013). If general onset 318 of senescence is similar among breeds, larger breeds will tend to have an abnormally shortened senior period, while 319 smaller breeds will likely undergo a protracted decline. Our findings indicate a similar pattern in cognitive 320 performance and are concordant with recent work finding similar prevalence of canine dementia across breeds of 321 varying size (Salvin et al. 2010, 2012). However, Kraus et al. (2013) suggest that large breeds physiologically 322 deteriorate rapidly, which we did not detect for cognitive aging. Together, these results may suggest that the 323 pathways which influence cognitive aging may be partially decoupled from those which affect the pace of 324 physiological aging. While further investigation is clearly warranted, these findings have important implications for 325 dog owners considering the quality of life during the senior period in terms of both cognitive and physiological 326 health.

327 We also observed effects of reproductive alteration on tasks involving responses to pointing gestures. Intact 328 dogs were more likely than spayed and neutered dogs to follow owners' cues across two tasks involving arm 329 pointing even after controlling for training history (Supplemental Table 7). These findings are consistent with results 
330 from a recent study that demonstrated reduced tendency of gonadectomized female dogs to follow human pointing 331 gestures compared to intact females (Scandurra et al. 2019). Neutering increases food motivation and decreases

332 metabolic rate, which can lead to lower energy levels and increased risk of obesity (Duffy and Serpell 2006; German 333 2006). It is therefore possible that neutered dogs had a greater motivation to obtain the food than intact dogs and 334 were less attentive to the cue given by the owner. It is unclear how sex hormones alter cognition and behavior in 335 dogs, however, there is some evidence that intact male dogs may exhibit slower cognitive decline than neutered 336 males (Hart 2001) which may be due to neuroprotective activity of sex hormones (Zárate et al. 2017). Additionally, 337 our findings demonstrated few and inconsistent sex-related differences in cognitive performance. Sex was 338 significantly associated with performance on two measures, duration of eye contact, in which male dogs held 339 slightly longer eye contact than females, and the 150 second delayed memory trial, in which males had a greater 340 propensity than females to remember treat location after this time delay (although this effect was not detected for the 341 sensitivity analyses with reduced sample size). Sex differences in dog cognition have been reported across a variety 342 of measures including looking times in violation of expectation tasks, gaze at human faces following oxytocin 343 administration, and speed and accuracy in spatial memory tasks (Müller et al. 2011; Nagasawa et al. 2015; Mongillo 344 et al. 2017). However, these studies found that female dogs tended to show longer looking times compared to 345 males, and better performance on spatial memory, effects in the opposite direction to those we observed. Given the 346 inconsistency across studies, it will be important for future research to assess the robustness of sex differences on 347 these measures, as well as specific factors that may determine the nature of these effects (Miller and Halpern 2014). 348 One limitation of this study stems from a lack of very old dogs in our sample. We had relatively few dogs 349 of very old age: dogs aged 11 and older comprised $5 \%$ of our dataset. We observed greater variation in cognitive 350 performance in older dogs, particularly among the oldest dogs in the study. For many tasks, the performance of the 351 oldest aged dogs in our study ranged greatly, often spanning the full spectrum of the dependent variable's range. The 352 relatively small number of very old dogs means that assessing cognitive performance in this group is more 353 susceptible to the influence of a small number of individuals. Future studies further establishing how cognition 354 changes and varies among this demographic would be valuable. We suspect that the limited number of very old dogs 355 may result from a selection bias in which owners of older, potentially highly impaired dogs, may have been less 356 likely to pursue participation in these activities. Thus, active recruitment of the oldest dogs will be an important 357 priority for future research. While collecting self-reported data from dog owners enabled the relatively large sample 
size of this study, we had variation in the reporting of information such as training history, which decreased power

359 to detect effects of aging and possible associations with mean breed lifespan within this group. It will be important

360 to generate larger datasets in the future that can include other potentially relevant covariates. We also collected data

361 from dog owners at a single time point which limited the degree to which we could evaluate individual variation in

362 cognitive performance throughout aging. Lastly, the cognitive assessment we used included diverse tasks, but the

363 particular cognitive processes measured by each specific task were not unambiguous.

364 Due to extraordinary intraspecific phenotypic diversity, dogs present a unique model for investigating how

365 age-related traits vary with cognition across the lifespan. Our findings suggest that age-related changes in executive

366 function in domestic dogs follow patterns similar to those in humans and provide insight regarding the relationships

367 (or lack thereof) between life history and cognitive trajectories in a species characterized by extensive intraspecific

368 diversity. An important priority for future work will be to determine whether dogs and humans share similar aging

369 trajectories in other cognitive domains such as long-term memory, cognitive processing speed, and episodic-like

370 memory. Using consistent and readily deployable cognitive assessments, such as the ones presented here, future

371 studies could evaluate longitudinal changes in cognitive performance of the same cohort of dogs across various

372 timepoints to gain a finer grained understanding of dog cognitive aging.

373

374 Acknowledgements We thank Laurie Santos, Richard Wrangham, and all members of the Dognition team for

375 helping create Dognition.com; the citizen scientists who contributed in collecting these data; Sarah Converse for

376 helpful insight with statistical questions; Daniel Promislow for valuable feedback and sharing data vital to this

377 project; Kenny Chiou, India Schneider-Crease, Corbin Johnson, Ian Dowsett, Sierra Sams, Lia Koklic, Grace De

378 Castro, and Matthew Harrington for their feedback on previous drafts.

Author Contributions MMW, ELM, BH, and NSM conceived of the project. MMW, ELM, and NSM analyzed the data. MMW, ELM, BH, JC, JK, ÁM, and NSM wrote the paper.

383 Funding This work was supported by the National Institute of Health grants R00AG051764, U19AG057377,

384 R01AG060931, R01HD097732. ÁM was supported by the National Brain Research Program (2017-1.2.1-NKP- 
2017-00002) and from the ELTE 576 Institutional Excellence Program supported by the National Research,

Development and Innovation Office (NKFIH-1157-8/2019-DT).

\section{Compliance with Ethical Standards}

Conflict of Interest BH is the founder of Dognition.com and is a member of the Dognition.com Scientific Advisory

Board along with JC, JK, and ÁM.

Data Accessibility The dataset analyzed during the current study is not publicly available due to third party

restrictions but is available from the corresponding author on reasonable request and permission of Canines Inc.

394 Supplementary Materials associated with this article are available online. Code used to complete the described

395 analyses is available at https://github.com/mwatowich/Dog-cognition-across-aging.

\section{References}

Adams B, Chan A, Callahan H, Milgram NW (2000) The canine as a model of human cognitive aging. Prog NeuroPsychopharmacol \& Biol Psychiat 24:675-692

Akaike H (1974) A new look at the statistical model identification. IEEE Trans Automat Contr 19:716-723. doi: 10.1109/TAC.1974.1100705

Akdemir D, Okeke UG (2015) EMMREML: Fitting mixed models with known covariance structures. https://cran.rproject.org/package=EMMREML $\mathrm{R}$ package version 3.1

Alvarez JA, Emory E (2006) Executive function and the frontal lobes: A meta-analytic review. Neuropsychol Rev 16:17-42. doi: 10.1007/s11065-006-9002-x

Austad S. (2010) Animal size, metabolic rate, and survival, among and within species. In: Wolf NS (ed) The comparative biology of aging. Springer, Dordrecht, pp 27-41

Bartke A (2017) Somatic growth, aging, and longevity. npj Aging Mech Dis 3:1-6. doi: 10.1038/s41514-017-0014$\mathrm{y}$

Beas BS, Setlow B, Bizon JL (2013) Distinct manifestations of executive dysfunction in aged rats. Neurobiol Aging 34:2164-2174

Bizon JL, Woods AG (2009) Animal models of human cognitive aging. Humana Press

Chapagain D, Range F, Huber L, Virányi Z (2018) Cognitive aging in dogs. Gerontology 64:165-171. doi: $10.1159 / 000481621$

Chapagain D, Virányi Z, Wallis LJ, et al (2017) Aging of attentiveness in border collies and other pet dog breeds: The protective benefits of lifelong training. Front Aging Neurosci 9:1-14. doi: 10.3389/fnagi.2017.00100

Craik FIM, Bialystok E (2006) Cognition through the lifespan: Mechanisms of change. Trends Cogn Sci 10:131138. doi: $10.1016 /$ j.tics.2006.01.007

Diamond A (2013) Executive functions. Annu Rev Psychol 64:135-168. doi: 10.1146/annurev-psych-113011143750

Duffy DL, Serpell JA (2006) Center for the Interaction of Animals and Society, School of Veterinary Medicine, University of Pennsylvania Non-reproductive Effects of Spaying and Neutering on Behavior in Dogs Proceedings of the Third International Symposium on Non-Surgical Contracepti. In: Proceedings of the Third International Symposium on Non-Surgical Contraceptive Methods for Pet Population Control

Fan R, Olbricht G, Baker X, Hou C (2016) Birth mass is the key to understanding the negative correlation between lifespan and body size in dogs. Aging (Albany NY) 8:3209-3222. doi: 10.18632/aging.101081 
Fick LJ, Fick GH, Li Z, et al (2012) Telomere length correlates with life span of dog breeds. Cell Rep 2:1530-1536. doi: 10.1016/j.celrep.2012.11.021

Galis F, Van Der Sluij I, Van Dooren TJM, et al (2007) Do large dogs die young ? J Exp Zool (Molecular Dev Evol 308:119-126. doi: 10.1002/jez.b

German AJ (2006) The growing problem of obesity in dogs and cats. Am Soc Nutr 1940-1946

Harada CN, Love MCN, Triebel K (2013) Normal cognitive aging. Clin Geriatr Med 29:737-752. doi: 10.1016/j.cger.2013.07.002

Hare B (2017) Survival of the friendliest: Homo Sapiens evolved via selection for prosociality. Annu Rev Psychol 68:155-185. doi: 10.1146/annurev-psych-010416-044201

Hare B, Tomasello M (2005) Human-like social skills in dogs? Trends Cogn Sci 9:439-444. doi: $10.1016 /$ j.tics.2005.07.003

Harrison XA, Donaldson L, Correa-Cano ME, et al (2018) A brief introduction to mixed effects modelling and multi-model inference in ecology. PeerJ 1-32. doi: 10.7717/peerj.4794

Hart BL (2001) Effect of gonadectomy on subsequent development of age-related cognitive impairment in dogs. J Am Vet Med Assoc 219:51-56

Head E, William Milgram N, W.Cotman C (2001) Neurobiological models of aging in the dog and other vertebrate species. In: Functional Neurobiology of Aging. pp 457-468

Healy K, Guillerme T, Cooper N, et al (2014) Ecology and mode-of-life explain lifespan variation in birds and mammals. Proc R Soc B Biol Sci 281:20140298-20140298. doi: 10.1098/rspb.2014.0298

Hoffman JM, Creevy KE, Franks A, et al (2018) The companion dog as a model for human aging and mortality. Aging Cell 17:. doi: 10.1111/acel.12737

Horschler DJ, Hare B, Call J, et al (2019) Absolute brain size predicts dog breed differences in executive function. Anim Cogn. doi: 10.1007/s10071-018-01234-1

Jones P, Chase K, Martin A, et al (2008) Single-nucleotide-polymorphism-based association mapping of dog stereotypes. Genetics 179:1033-1044. doi: 10.1534/genetics.108.087866

Jurado MB, Rosselli M (2007) The elusive nature of executive functions: A review of our current understanding. Neuropsychol Rev 17:213-233. doi: 10.1007/s11065-007-9040-z

Kraus C, Pavard S, Promislow DEL (2013) The size-life span trade-off decomposed: Why large dogs die young. Am Nat 181:492-505. doi: 10.1086/669665

Lea AJ, Tung J, Zhou X (2015) A flexible, efficient binomial mixed model for identifying differential DNA methylation in bisulfite sequencing data. PLoS Genet 11:1-31. doi: 10.1371/journal.pgen.1005650

MacLean EL, Herrmann E, Suchindran S, Hare B (2017) Individual differences in cooperative communicative skills are more similar between dogs and humans than chimpanzees. Anim Behav 126:41-51. doi: 10.1016/j.anbehav.2017.01.005

Manrique HM, Call J (2015) Age-dependent cognitive in flexibility in great apes. Anim Behav 102:1-6. doi: 10.1016/j.anbehav.2015.01.002

Metcalfe NB, Monaghan P (2003) Growth versus lifespan : perspectives from evolutionary ecology. 38:935-940. doi: 10.1016/S0531-5565(03)00159-1

Miklósi Á, Topál J, Csányi V (2004) Comparative social cognition: What can dogs teach us? Anim Behav 67:9951004. doi: 10.1016/j.anbehav.2003.10.008

Milgram NW, Head E, Weiner E, Thomas E (1994) Cognitive functions and aging in the dog: acquisition of nonspatial visual tasks. Behav Neurosci 108:57-68. doi: https://doi.org/10.1037/0735-7044.108.1.57

Miller DI, Halpern DF (2014) The new science of cognitive sex differences. Trends Cogn Sci 18:37-45. doi: 10.1016/j.tics.2013.10.011

Mongillo P, Scandurra A, Aniello BD, Marinelli L (2017) Effect of sex and gonadectomy on dogs' spatial performance. Appl Anim Behav Sci 191:84-89. doi: 10.1016/j.applanim.2017.01.017

Moore TL, Killiany RJ, Herndon JG, et al (2006) Executive system dysfunction occurs as early as middle-age in the rhesus monkey. Neurobiol Aging 27:1484-1493. doi: 10.1016/j.neurobiolaging.2005.08.004

Müller CA, Mayer C, Dörrenberg S, et al (2011) Female but not male dogs respond to a size constancy violation. Biol Lett 689-691. doi: 10.1098/rsbl.2011.0287

Nagasawa M, Mitsui S, En S, et al (2015) Oxytocin-gaze positive loop and the coevolution of human-dog bonds. Sci Reports 348:

Ostrander EA, Wayne RK, Freedman AH, Davis BW (2017) Demographic history, selection and functional diversity of the canine genome. Nat Rev Genet 18:705-720. doi: 10.1038/nrg.2017.67

Parker HG, Dreger DL, Rimbault M, et al (2017) Genomic analyses reveal the influence of geographic origin, migration, and hybridization on modern dog breed development. Cell Rep 19:697-708. doi: 
10.1016/j.celrep.2017.03.079

Rodefer JS, Nguyen TN (2008) Naltrexone reverses age-induced cognitive deficits in rats. Neurobiol Aging 29:309313. doi: 10.1016/j.neurobiolaging.2006.10.005

Salvin HE, Mcgreevy PD, Sachdev PS, Valenzuela MJ (2010) Under diagnosis of canine cognitive dysfunction: A cross-sectional survey of older companion dogs. Vet J 184:277-281. doi: 10.1016/j.tvj1.2009.11.007

Salvin HE, McGreevy PD, Sachdev PS, Valenzuela MJ (2012) The effect of breed on age-related changes in behavior and disease prevalence in cognitively normal older community dogs, Canis lupus familiaris. J Vet Behav 7:61-69

Scandurra A, Alterisio A, Di Cosmo A, et al (2019) Ovariectomy impairs socio-cognitive functions in dogs. Animals 9:. doi: 10.3390/ani9020058

Stewart L, Rodriguez K, Call J, et al (2015) Citizen science as a new tool in dog cognition research. PLoS One 10:e135176. doi: 10.1371/journal.pone.0135176

Sun S, Zhu J, Mozaffari S, et al (2019) Heritability estimation and differential analysis of count data with generalized linear mixed models in genomic sequencing studies. Bioinformatics 35:487-496. doi: 10.1093/bioinformatics/bty644

Szabó D, Gee NR, Miklósi Á (2016) Natural or pathologic? Discrepancies in the study of behavioral and cognitive signs in aging family dogs. J Vet Behav Clin Appl Res 11:86-98. doi: 10.1016/j.jveb.2015.08.003

Tapp PD, Siwak CT, Estrada J, et al (2003) Size and reversal learning in the beagle dog as a measure of executive function and inhibitory control in aging. Learn Mem 10:64-73. doi: 10.1101/1m.54403

Verhaeghen P (2011) Aging and executive control: Reports of a demise greatly exaggerated. Curr Dir Psychol Sci 20:174-180. doi: 10.1177/0963721411408772

VonHoldt BM, Pollinger JP, Lohmueller KE, et al (2010) Genome-wide SNP and haplotype analyses reveal a rich history underlying dog domestication. Nature 464:898-902. doi: 10.1038/nature08837

Wallis LJ, Range F, Müller CA, et al (2014) Lifespan development of attentiveness in domestic dogs: Drawing parallels with humans. Front Psychol 5:. doi: 10.3389/fpsyg.2014.00071

Wallis LJ, Virányi Z, Müller CA, et al (2016) Aging effects on discrimination learning, logical reasoning and memory in pet dogs. Age (Omaha) 38:1-18. doi: 10.1007/s11357-015-9866-x

Zárate S, Stevnsner T, Gredilla R (2017) Role of estrogen and other sex hormones in brain aging. Neuroprotection and DNA repair. Front Aging Neurosci 9:1-22. doi: 10.3389/fnagi.2017.00430 